\title{
Lebensqualität und körperliche Leistungsfähigkeit bei Patienten nach Lungentransplantation
}

\author{
U. Tegtbur ${ }^{1}$ \\ C. Sievers ${ }^{1}$ \\ M. W. Busse ${ }^{2}$ \\ K. Pethig ${ }^{3}$ \\ G. Warnecke ${ }^{4}$ \\ C. Kugler ${ }^{4}$ \\ E. Gützlaff 1 \\ H. W. Künsebeck ${ }^{5}$ \\ M. Strüber ${ }^{4}$ \\ A. Haverich ${ }^{4}$ \\ J. Niedermeyer 6
}

\author{
Quality of Life and Exercise Capacity in Lung Transplant Recipients
}

\section{Zusammenfassung}

Hintergrund: Patienten nach Lungentransplantation (LTX) bewerten ihre Lebensqualität (LQ) nahezu gleich gut wie Gesunde. Dagegen zeigten andere Untersuchungen, dass die körperliche Leistungsfähigkeit nach LTX auf 30-40\% der Normwerte verringert bleibt. Ziel dieser Studie war es, die Diskrepanz zwischen reduzierter Leistungsfähigkeit und subjektiv empfundener guter Lebensqualität in einem Kollektiv Lungentransplantierter zu untersuchen, mögliche Zusammenhänge zu beschreiben und die Resultate mit denen einer Kontrollgruppe zu vergleichen. Methode: 27 Patienten $208 \pm 67$ Tage nach bilateraler LTX ( $16 \mathrm{~m}$, $11 \mathrm{w}$; Alter $46 \pm 10$ Jahre; Bodymass-Index $24 \pm 3 \mathrm{~kg} \cdot \mathrm{m}^{-2}$, $\mathrm{FEV}_{1} \%$ $75 \pm 27 \%$ ) und 30 Probanden der Kontrollgruppe (KG; $17 \mathrm{~m}, 13 \mathrm{w}$; Alter $47 \pm 15$ Jahre; BMI $26 \pm 4 \mathrm{~kg} \cdot \mathrm{m}^{-2}$, $\mathrm{FEV}_{1} \% 103 \pm 15 \%$ ) wurden mit einem ergospirometrischen Stufentest sowie dem Fragebogen „Profil der Lebensqualität chronisch Kranker“ untersucht. Ergebnisse: Die Lungentransplantierten zeigten im Stufentest eine verminderte maximale Sauerstoffaufnahme (LTX 15,1 \pm $\left.1,8 \mathrm{ml} \cdot \mathrm{min}^{-1} \cdot \mathrm{kg}^{-1} ; \mathrm{KG} 34,5 \pm 9,1 \mathrm{ml} \cdot \mathrm{min}^{-1} \cdot \mathrm{kg}^{-1} ; \mathrm{p}<0,01\right)$, eine reduzierte maximale Leistung (LTX $1,0 \pm 0,2 \cdot \mathrm{kg}^{-1}$; KG $2,4 \pm$ $\left.1,0 \mathrm{~W} \cdot \mathrm{kg}^{-1} ; \mathrm{p}<0,01\right)$ und eine reduzierte altersbezogene Soll-

\section{Abstract}

Background: Quality of life in lung transplant recipients (LTR) is reported to be comparable with that of the general population. However, previous studies have shown that exercise capacity was reduced to $30-40 \%$ of normal values. The purpose of this study was to investigate the gap between good self-reported quality of life and reduced exercise capacity in LTR, to describe possible correlations and to compare the results with those of a control group (CG). Methods: 27 LTR $208 \pm 67$ days after bilateral lund transplantation (16 male, 11 female; age: $46 \pm 10$ years; body mass index: $24 \pm 3 \mathrm{~kg} \cdot \mathrm{m}^{-2}, \mathrm{FEV}_{1} \% 75 \pm 27 \%$ ) and 30 controls (17 male 13 female; age $47 \pm 15$ years; BMI: $26 \pm 4 \mathrm{~kg} \cdot \mathrm{m}^{-2}$, $\mathrm{FEV}_{1} \% 103 \pm 15 \%$ ) performed cardiopulmonary exercise testing and were interviewed with the standardized German „Quality of life profile for chronic disease" self-rating questionnaire. Results: Significant differences were shown in objective exercise related variables (peak oxygen consumption: LTR $15.1 \pm 1.8$, CG $\left.34.5 \pm 9.1 \mathrm{ml} \cdot \mathrm{min}^{-1} \cdot \mathrm{kg}^{-1} ; \mathrm{p}<0,01\right)$; peak workload: LTR $1.0 \pm$ 0.2 ; CG $2.4 \pm 1.0 \mathrm{~W} \cdot \mathrm{kg}^{-1} ; \mathrm{p}<0.01$ ); percentage of predicted workload: LTR $44 \pm 12$, CG $115 \pm 33 \%$; $<<0.01$ ). The rating of subjective quality of life in physical, psychological and social domains

Institutsangaben

${ }^{1}$ Medizinische Hochschule Hannover, Sportmedizinisches Zentrum (Leiter: Dr. U. Tegtbur)

${ }^{2}$ Universität Leipzig, Institut für Sportmedizin (Direktor Prof. Dr. M. W. Busse)

${ }^{3}$ Friedrich Schiller Universität Jena, Klinik für Innere Medizin III - Kardiologie

(Direktor: Prof. Dr. H. R. Figulla)

${ }^{4}$ Medizinische Hochschule Hannover, Abteilung Thorax-, Herz- und Gefäßchirurgie

(Direktor: Prof. Dr. A. Haverich)

${ }^{5}$ Medizinische Hochschule Hannover, Abteilung Psychosomatik und Psychotherapie

(Direktor: Prof. Dr. F. Lamprecht)

${ }^{6}$ Medizinische Hochschule Hannover, Abteilung Pneumologie

(Komm. Leiter Dr. J. Niedermeyer)

Danksagung

Für die Untersuchungen haben wir eine finanzielle Unterstützung von Hoffman-La Roche erhalten.

Dafür bedanken wir uns sehr.

Korrespondenzadresse

Dr. med. Uwe Tegtbur · Medizinische Hochschule Hannover · Sportmedizinisches Zentrum (OE 4252).

Carl-Neuberg-Str. 1 · 30625 Hannover · E-mail: tegtbur.uwe@mh-hannover.de

Eingang: 31. Juli 2003 · Nach Revision angenommen: 30. Oktober 2003

Bibliografie

Pneumologie 2004; 58: 72-78 @ Georg Thieme Verlag Stuttgart · New York .

ISSN 0934-8387 · DOI 10.1055/s-2003-812526 
leistung (LTX $44 \pm 12 \%$, KG $115 \pm 33 \%$; $p<0,01$ ). Die LTX und die KG bewerteten ihre LQ in der physischen, sozialen und psychischen Dimension jeweils gleich gut. Die Bewertung der LQ der LTX entsprach dabei auch den Mittelwerten der Population der Fragebogennormierung $(n=1143)$. Die Pearson-Korrelation ergab eine positiv signifikante Beziehung zwischen subjektiver physischer LQ und der körperlichen Leistungsfähigkeit (LTX r $=0,44, p<0,05 ; K G r=0,37, p<0,05)$. Schlussfolgerung: Patienten 7 Monate nach LTX beschrieben ihre subjektive LQ so gut wie gesunde Kontrollpersonen. Dagegen ist die körperliche Leistungsfähigkeit bei guter Transplantatfunktion erheblich reduziert. Damit Alltagsbelastungen besser toleriert werden können, sollte ein körperliches Trainingsprogramm in die Nachsorge Lungentransplantierter eingebunden werden. of LTR did not differ from values of the CG or of the general population ( $\mathrm{n}=1143$ ). The quality of life in the physical domain correlated significantly with peak exercise capacity $($ LTR $r=0.44$, $\mathrm{p}<0.05 ; \mathrm{CG} r=0.37$; $\mathrm{p} 0.05$ ). Conclusion: Patients 7 months after lung transplantation described their physical, social and psychological quality of life as equally good as the healthy control group. However, peak exercise capacity and oxygen consumption were markedly reduced. To improve physical capacity in the range of daily activities, an exercise training program should be offered to patients after lung transplantation.

\section{Einleitung}

Die Lungentransplantation hat sich in der Therapie von Patienten mit fortgeschrittenem Lungenversagen etabliert. Bisher wurden weltweit mehr als 16000 Lungentransplantationen durchgeführt. Die aktuarischen 1- bzw. 5-Jahres-Überlebensraten konnten durch standardisierte und effektive Nachsorgeprogramme auf 73 bzw. $45 \%$ gesteigert werden [1]. Neben der medizinischen Nachsorge nimmt auch das Interesse an einer Verbesserung der Lebensqualität, der körperlichen Funktion und der Leistungsfähigkeit zu.

Nach Lungentransplantation (LTX) ist die selbsteingeschätzte Lebensqualität erheblich verbessert und erreicht nahezu Normalwerte [2]. Patienten 7 Monate nach Lungentransplantation gaben an, dass körperliche Aktivitäten wie Treppensteigen, Walking oder Hausarbeit nicht mehr bzw. nur noch in bis zu $12 \%$ der Fälle mit stärkerer gefühlter Anstrengung verbunden waren [3].

Demgegenüber bleiben objektivierbare Befunde der Lebensqualität nach Lungentransplantation wie z.B. die körperliche Leistungsfähigkeit deutlich hinter Normalwerten zurück. Nach Lungentransplantation konnte eine signifikante Verbesserung der maximalen Sauerstoffaufnahme gemessen werden (von $11 \mathrm{ml} \cdot \mathrm{min}^{-1} \cdot \mathrm{kg}^{-1}$ vor LTX auf $14 \mathrm{ml} \cdot \mathrm{min}^{-1} \cdot \mathrm{kg}^{-1} 2$ Monate nach LTX) [4]. Auch 2 bis 3 Jahre nach einseitiger- oder bilateraler Lungentransplantation bleibt die körperliche Leistungsfähigkeit auf 38 bzw. 37\% des Sollwertes Gesunder reduziert [5]. Die eingeschränkte Funktion der Skelettmuskulatur wird als die wesentliche Ursache der verminderten Leistungsfähigkeit beschrieben $[5,6]$.

Bisher liegt keine Studie bei Patienten nach Lungentransplantation vor, in der sowohl die subjektiv eingeschätzte Lebensqualität als auch die objektive körperliche Leistungsfähigkeit erfasst wurden [7].

Daher war es Ziel der Studie, die gegensätzlichen Befunde früherer Studien mit der einerseits als gut beschriebenen subjektiven Lebensqualität und der andererseits als objektiv erheblich reduziert gemessenen körperlichen Leistungsfähigkeit aufzuarbeiten und zu vergleichen. Bei Patienten im ersten Jahr nach Lungen- transplantation wurde hierzu die körperliche Leistungsfähigkeit in ergospirometrischen Stufentests untersucht sowie die Lebensqualität aus Sicht der Patienten mit standardisierten Fragebogen evaluiert.

\section{Methodik}

60 Patienten erhielten zwischen Juni 2001 und September 2002 eine bilaterale Lungentransplantation an der Medizinischen Hochschule Hannover. Weitere Einschlusskriterien für die Teilnahme an der Untersuchung waren: Alter 18 bis 60 Jahre, stabiler hämodynamischer und respiratorischer Zustand, schriftliche Einwilligung. Ausschlusskriterien waren: Schwere akute Transplantatabstoßung, Bronchiolitis obliterans Syndrom, Infektion oder Besiedlung mit multiresistenten Keimen, Begleiterkrankungen als Kontraindikation für körperliche Belastung, orthopädische Einschränkung der Ergometrie.

Von den 60 Patienten verstarben 9 in den ersten 8 Monaten nach Transplantation. 14 Patienten nahmen wegen persönlicher Gründe (Ablehnung, weite Anreise) nicht an der Studie teil. Bei 10 Patienten lagen medizinische Kontraindikationen vor (AbstoBungsreaktion $n=2$; Bronchusstenose $n=2$; multiresistente Keime $n=3$; Gelenkarthrose $n=2$; schwere gastrointestinale Erkrankung $n=1$ ). Bei 27 Patienten konnte $7 \pm 2$ Monate nach Lungentransplantation das Untersuchungsprogramm komplett abgeschlossen werden, und nur diese wurden in die weitere Auswertung aufgenommen. Die Indikationen zur Lungentransplantation waren: Lungenfibrose $(\mathrm{n}=7) ; \alpha$-1-Antitrypsinmangel $(n=3)$; Lungenemphysem bei COPD $(n=4)$; zystische Fibrose $(n=5)$; Bronchiektasen $(n=3)$; Sarkoidose $(n=2)$; Lymphangioleiomyomatose $(n=1)$; akute interstitielle Pneumonie $(n=1)$; Eisenmenger-Syndrom $(n=1)$. Patienten mit einer akuten AbstoBungsreaktion wurden ausgeschlossen. Alle Patienten erhielten postoperativ eine dreifache Immunsuppression bestehend aus Cyclosporin A ( $\mathrm{n}=23$ ) oder Tacrolimus $(\mathrm{n}=4)$, Mycophenolatmofetil $(n=25)$ oder Sirolimus $(n=2)$, und Prednisolon $(n=27)$. Eine antiinfektiöse Prophylaxe wurde mit Aciclovir und Cotrimoxazol bei allen Patienten durchgeführt. Die weitere medikamentöse Therapie bestand aus ACE-Hemmern $(\mathrm{n}=2), \mathrm{AT}_{2}$-Antagonisten $(n=2)$, Kalzium-Antagonisten $(n=5), \beta$-Blockern $(n=10)$, Diuretika $(\mathrm{n}=18)$ und Statinpräparaten $(\mathrm{n}=4)$. 
Kontrollgruppe: Die Kontrollgruppe (KG) wurde aus 30 gesunden, untrainierten Probanden gebildet, die eine vergleichbare Alters- und Geschlechterverteilung wie die Gruppe der Lungentransplantierten zeigten. Die Probanden haben sich aus eigener Motivation und in konsekutiver Reihenfolge in der sportmedizinischen Ambulanz zur Einleitung eines allgemein-präventiven Sportprogramms vorgestellt. Einschlusskriterien für die Aufnahme in die Kontrollgruppe waren: Alter 18 bis 60 Jahre, schriftliche Einwilligung. Ausschlusskriterien waren: Regelmäßiges körperliches Ausdauertraining in den letzten 12 Monaten vor der Untersuchung, kardiale oder pulmonale Erkrankungen, orthopädische Einschränkungen oder Kontraindikation der Ergometrie. Alle Probanden beendeten die Untersuchungsteile und wurden in die Auswertung aufgenommen. Die Untersuchung wurde von der Ethikkommission der Medizinischen Hochschule Hannover genehmigt.

\section{Spiroergometrie und Funktionsdiagnostik}

Bei jedem Patienten wurde vor der Ergospirometrie eine Anamnese, eine körperliche Untersuchung, eine Lungenfunktionsmessung und eine Messung des Körperfettanteils mit der 10-PunkteCaliper-Methode nach Parízková durchgeführt [8]. In einer maximalen bzw. symptomlimitierten Ergospirometrie (Ergoline 900, Ergoline, Bitz) wurden Herzfrequenz und Atemgase kontinuierlich (Oxycon Delta, Jäger, Würzburg), Blutdruck und Blutlaktatkonzentration alle 3 min gemessen (Ebio 6666, Eppendorf, Hamburg).

\section{Subjektive Einschätzung der Lebensqualität}

Die Erhebung der subjektiven Lebensqualität erfolgte mit dem von Siegrist u. Mitarb. entwickelten „Profil der Lebensqualität chronisch Kranker“ (PLC) [9]. Die Patienten beantworteten nach standardisierter schriftlicher Anleitung 40 Likert-skalierte Fragen zur gesundheitsbezogenen Lebensqualität im Selbst-rating. Zur Auswertung sind die Antworten von 0 bis 4 skaliert, wobei ein höherer Summenscore in allen Skalen jeweils einer besseren Lebensqualität entspricht.

Die 40 Items des PLC sind in 6 Skalen zusammengefasst und können den 3 Dimensionen der Lebensqualität zugeordnet werden: 1. Physische Dimension mit der Skala „Subjektives Leistungsvermögen" (körperliche und geistige Funktionsfähigkeit im privaten und beruflichen Alltag); 2. psychische Dimension mit den Skalen „Genuss- und Entspannungsfähigkeit“ (individuelle Fähigkeit der psychischen Regeneration), „Positive Stimmung“ (Aufmerksamkeit, gute Laune, Ausgeglichenheit und Zuversicht) und „Negative Stimmung“ (Skala umgepolt; Niedergeschlagenheit, Nervosität, Reizbarkeit, Bedrohungsgefühle und Hoffnungslosigkeit); 3. soziale Dimension mit den Skalen „Kontaktvermögen“ (Fähigkeit, menschliche Beziehungen aufzubauen und zu erhalten) und „Zugehörigkeitsgefühl“ (sozio-emotionaler Rückhalt mit Nähe, Zuwendung und Hilfeleistung) $[9,10]$.

\section{Statistik}

Alle Werte werden als Mittelwerte mit Standardabweichung angegeben. Die Unterschiede zwischen den Gruppen in den Lebensqualitätsskalen und den funktionalen Variablen wurden mit unabhängigen, zweiseitigen t-Tests untersucht. Die Beziehung zwischen den objektiven Variablen und den Skalen des PLC-Fragebogens wurde nach Überprüfung auf Normalvertei- lung mit der zweiseitigen Korrelation nach Pearson berechnet. Mit der multiplen Korrelationsanalyse wurde der Einfluss der objektiven, unabhängigen Variablen auf die subjektiven, abhängigen Variablen quantifiziert (schrittweise Regression mit Vorwärtstechnik und Schrumpfkorrektur). $\mathrm{p}<0,05$ galt als signifikant, $p<0,01$ als hochsignifikant. Die statistische Analyse erfolgte mit SPSS Vs. 11.0.

\section{Ergebnisse}

Die Patientencharakteristika sind in Tab.1 dargestellt. Die Kontrollgruppe war signifikant schwerer als die lungentransplantierten Patienten. Da die körperliche Leistungsfähigkeit und die maximale Sauerstoffaufnahme gewichtsabhängige Größen sind, wurden zur Beurteilung der objektiven Leistungsfähigkeit gewichtsbezogene Parameter und die alters-, geschlechts- und gewichtsnormierte prozentual erreichte Sollleistung angegeben.

Tab. 1 Patientencharakteristika

\begin{tabular}{|c|c|c|}
\hline & $\operatorname{LTX}(n=27)$ & $K G(n=30)$ \\
\hline Geschlecht & $11 \mathrm{w} 16 \mathrm{~m}$ & $13 w 17 m$ \\
\hline Alter (Jahre) & $46 \pm 10$ & $47 \pm 15$ \\
\hline Größe (cm) & $169 \pm 9$ & $174 \pm 9$ \\
\hline Gewicht (kg) & $68 \pm 14$ & $80 \pm 11^{* *}$ \\
\hline$B M I\left(\mathrm{~kg} \cdot \mathrm{m}^{-2}\right)$ & $23,6 \pm 3,4$ & $26,5 \pm 3,9^{* *}$ \\
\hline Körperfett (\%) & $20,6 \pm 4,8$ & $24,4 \pm 7,7^{*}$ \\
\hline$H_{\text {Ruhe }}\left(S \cdot \min ^{-1}\right)$ & $94 \pm 17$ & $82 \pm 14^{* *}$ \\
\hline$R R_{\text {sysRuhe }}(\mathrm{mm} \mathrm{Hg})$ & $132 \pm 17$ & $127 \pm 12$ \\
\hline$R R_{\text {diaRuhe }}(\mathrm{mm} \mathrm{Hg})$ & $92 \pm 12$ & $91 \pm 8$ \\
\hline$V C(I)$ & $3,20 \pm 1,02$ & $4,21 \pm 1,25^{* *}$ \\
\hline VC \% vom Soll & $79.3 \pm 24,3$ & $98,5 \pm 13,6^{* *}$ \\
\hline$F E V_{1}(I)$ & $2,43 \pm 0,82$ & $3,72 \pm 1,51^{* *}$ \\
\hline $\mathrm{FEV}_{1} \%$ vom Soll & $74,8 \pm 26,7$ & $102,7 \pm 15,2^{* *}$ \\
\hline
\end{tabular}

LTX, Patienten nach Lungentransplantation; KG, Kontrollgruppe. BMI, Bodymass-Index. HF, Herzfrequenz. RR, Blutdruck. VC, Vitalkapazität. $\mathrm{FEV}_{1}$, Einsekundenkapazität.

${ }^{*} \mathrm{p}<0,05 ;{ }^{* *} \mathrm{p}<0,01$ LTX vs. KG

Die Vitalkapazität und die Einsekundenkapazität der Lungentransplantierten waren auf $79 \pm 24 \%$ bzw. $75 \pm 27 \%$ der Sollwerte reduziert, während die Kontrollgruppe mit $99 \pm 14 \%(\mathrm{p}<0,01)$ bzw. $103 \pm 15 \%(p<0,01)$ Normalwerte erreichte.

\section{Spiroergometrie und Funktionsdiagnostik}

Alle spiroergometrischen Stufentests wurden wegen Dyspnoe oder periphermuskulärer Erschöpfung beendet. Das subjektive Belastungsempfinden bei Testabbruch wurde in beiden Gruppen mit schwer bis sehr schwer nach der Borg-Skala eingeschätzt [11]. Die lungentransplantierten Patienten erreichten mit $44 \pm$ $12 \%$ eine signifikant schlechtere Sollleistung als die Kontrollgruppe mit $115 \pm 33 \%$ ( $\mathrm{p}<0,01$; siehe Tab. 2 ). Die absolute Leistung und die Maximalwerte von Herzfrequenz, Laktat und Sauerstoffaufnahme waren bei den Lungentransplantierten signifikant niedriger als in der Kontrollgruppe. 
Tab. 2 Kardiopulmonale Funktionsdiagnostik. Dargestellt sind Mittelwerte \pm SD

\begin{tabular}{|c|c|c|}
\hline & $\operatorname{LTX}(n=27)$ & $K G(n=30)$ \\
\hline erreichte Sollleistung (\%) & $44 \pm 12$ & $115 \pm 33^{*}$ \\
\hline maximale Leistung $(W)$ & $65 \pm 17$ & $195 \pm 72^{*}$ \\
\hline$V_{2 \max }\left(\mathrm{ml} \cdot \mathrm{min}^{-1} \cdot \mathrm{kg}^{-1}\right)$ & $15,1 \pm 1,8$ & $34,5 \pm 9,1^{*}$ \\
\hline$H F_{\max }\left(S \cdot \min ^{-1}\right)$ & $128 \pm 17$ & $168 \pm 20^{*}$ \\
\hline$R R_{\text {sys max }}(\mathrm{mm} \mathrm{Hg})$ & $184 \pm 29$ & $205 \pm 28^{*}$ \\
\hline$R R_{\text {dia max }}(\mathrm{mm} \mathrm{Hg})$ & $98 \pm 10$ & $101 \pm 12$ \\
\hline Laktat $_{\max }\left(\mathrm{mmol} \cdot \mathrm{I}^{-1}\right)$ & $5,4 \pm 1,1$ & $8,4 \pm 2,3^{*}$ \\
\hline Borg-Skala (Einheiten) & $15,0 \pm 1,6$ & $16,2 \pm 1,6^{*}$ \\
\hline $\operatorname{HRR}\left(\mathrm{S} \cdot \mathrm{min}^{-1}\right)$ & $33 \pm 12$ & $85 \pm 20^{*}$ \\
\hline
\end{tabular}

LTX, Patienten nach Lungentransplantation; KG, Kontrollgruppe.

$\mathrm{VO}_{2 \max }$, maximale Sauerstoffaufnahme. HF, Herzfrequenz. RR, Blutdruck. HRR, Herzfrequenzreserve. ${ }^{*} \mathrm{p}<0,01$ LTX vs. KG.

\section{Subjektive Einschätzung der Lebensqualität}

Abb.1 zeigt die Ergebnisse der standardisierten Befragung mit dem „Profil der Lebensqualität chronisch Kranker“. In keiner der 6 Skalen der selbsteingeschätzten Lebensqualität ergaben sich signifikante Unterschiede zwischen Lungentransplantierten und den Probanden der Kontrollgruppe. Im Vergleich zur repräsentativen Stichprobe zur Normierung der Skalen $(n=1143$ bis 1273) waren die Werte der Lungentransplantierten in 5 von 6 Skalen tendenziell besser [12]. Nur die Einschätzung in der Skala „Subjektives Leistungsvermögen“ als Parameter der körperlichen und geistigen Funktionsfähigkeit im Alltag wurde von den Lungentransplantierten geringfügig schlechter bewertet als von der Normstichprobe (2,66 $\pm 0,66$ vs. $2,70 \pm 0,62$ Einheiten).

\section{Korrelationen von subjektiver Lebensqualität mit objektiven Messgrößen}

Die Beziehungen zwischen den objektiven Funktionsparametern und der aus Sicht der Probanden berichteten Lebensqualität in den 6 Skalen des PLC wurden für Lungentransplantierte und die Kontrollgruppe analysiert und in Tab. 3 dargestellt.
Die von den Lungentransplantierten bewertete Lebensqualität in der physischen Dimension (Skala „subjektives Leistungsvermögen“) korrelierte signifikant positiv mit der maximalen körperlichen Leistungsfähigkeit und auch mit der Einsekundenkapazität. Zwischen den Skalen der psychischen bzw. der sozialen Dimension der subjektiven Lebensqualität und der objektiven Maximalleistung bzw. der $\mathrm{FEV}_{1} \%$ fanden sich keine signifikanten Korrelationen. Das Alter der lungentransplantierten Patienten zeigte eine siginifkant negative Beziehung zur Selbsteinschätzung in der Skala „Genuss- und Entspannungsfähigkeit“. Die Lebensqualitätsskalen 1 bis 6 korrelierten in beiden Gruppen signifikant positiv miteinander ( $\mathrm{r}$ zwischen 0,35 und 0,78 ).

Mit der multiplen Korrelationsanalyse wurde der Einfluss von Alter, BMI, Körperfettanteil, Maximalleistung, prozentual erreichter Solleistung, maximaler Sauerstoffaufnahme, Herzfrequenz, $\mathrm{FEV}_{1} \%$ und maximaler Blutlaktatkonzentration auf jede der sechs Skalen der Lebensqualität schrittweise untersucht.

Die Lebensqualität in der Skala „subjektives Leistungsvermögen“ (entsprechend der physischen Dimension) konnte bei den Lungentransplantierten zu 19\% durch die gewichtsbezogene Maximalleistung $(p<0,05)$ vorhergesagt werden. Die anderen Größen führten nicht zu einer weiteren signifikanten Verbesserung des Vorhersagepotentials. Der Einfluss der objektiven Variablen auf die psychischen und sozialen Dimensionen der Lebensqualität ließ sich nicht signifikant gewichten.

\section{Diskussion}

Bei Patienten im Mittel 7 Monate nach Lungentransplantation und bei einer gesunden Kontrollgruppe wurden die subjektiv eingeschätzte Lebensqualität und die objektive körperliche Leistungsfähigkeit untersucht und vergleichend gegenübergestellt.

Hauptresultate der Untersuchung waren 1.) eine Reduktion der objektiven körperlichen Leistungsfähigkeit bei den Lungentransplantierten auf durchschnittlich $44 \%$ des Normalwertes, 2.) eine gleich gute oder nur tendenziell verringerte Selbsteinschätzung in allen Skalen der Lebensqualität der Lungentransplantierten

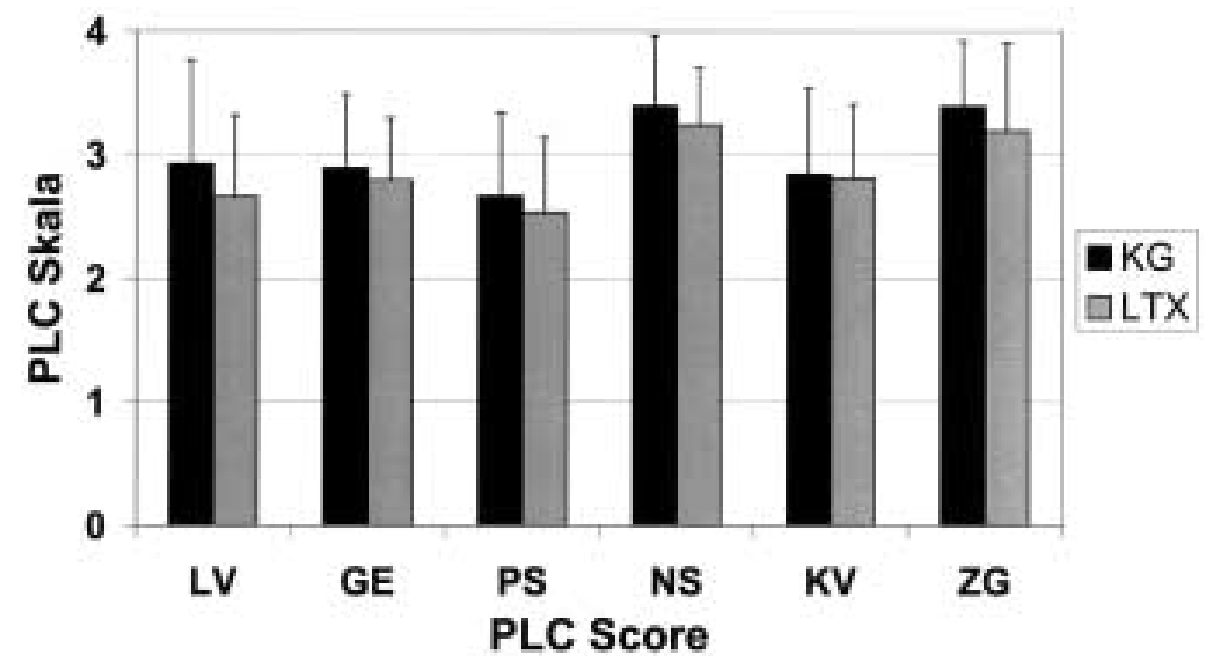

Abb. 1 Subjektive Einschätzung der Lebensqualität von Patienten $7 \pm 2$ Monate nach Lungentransplantation (LTX, $\mathrm{n}=27$ ) und der Kontrollgruppe (KG, $n=30)$. Dargestellt sind Mittelwerte \pm SD in den 6 Skalen des Fragebogens „Profil der Lebensqualität chronisch Kranker“ (PLC): Selbst eingeschätztes Leistungsvermögen (LV), Genussund Entspannungsfähigkeit (GE), positive Stimmung (PS), negative Stimmung (NS), Kontaktvermögen (KV) und Zugehörigkeitsgefühl (ZG). Ein höherer Wert zeigt jeweils eine bessere Selbsteinschätzung der Lebensqualität an. Es finden sich keine signifikanten Unterschiede zwischen den beiden Gruppen. 
Tab. 3 Beziehung zwischen subjektiven Lebensqualitätsskalen, Funktionsparametern sowie Patientencharakteristika für LTX und KG, berechnet mit der Pearson-Korrelation

\begin{tabular}{|c|c|c|c|c|c|c|c|}
\hline \multirow[t]{6}{*}{ LTX } & erreichte Sollleistung & 0,35 & $-0,01$ & 0,10 & 0,13 & 0,13 & 0,15 \\
\hline & maximale Leistung $\cdot \mathrm{kg}^{-1}$ & $0,44^{\mathrm{a}}$ & 0,18 & 0,11 & 0,13 & 0,11 & 0,06 \\
\hline & Körperfett & $-0,08$ & $-0,08$ & $-0,11$ & $-0,04$ & $-0,16$ & 0,12 \\
\hline & VC \% vom Soll & 0,38 & 0,24 & 0,27 & 0,04 & 0,28 & 0,21 \\
\hline & $\mathrm{FEV}_{1} \%$ vom Soll & $0,40^{\mathrm{a}}$ & 0,16 & 0,19 & 0,10 & 0,21 & 0,20 \\
\hline & $\mathrm{m} / \mathrm{w}$ & 0,34 & 0,09 & 0,27 & 0,18 & 0,34 & 0,24 \\
\hline \multirow[t]{4}{*}{ KG } & erreichte Sollleistung & $0,37^{\mathrm{a}}$ & 0,00 & $-0,05$ & $-0,30$ & $-0,03$ & $-0,36$ \\
\hline & maximale Leistung $\cdot \mathrm{kg}^{-1}$ ) & $0,37^{\mathrm{a}}$ & $-0,01$ & $-0,01$ & $-0,32$ & $-0,11$ & $-0,14$ \\
\hline & $\mathrm{FEV}_{1} \%$ vom Soll & 0,05 & 0,02 & $-0,07$ & $-0,19$ & 0,21 & $-0,08$ \\
\hline & $\mathrm{m} / \mathrm{w}$ & $-0,28$ & $-0,07$ & $-0,07$ & 0,18 & $-0,14$ & $-0,02$ \\
\hline
\end{tabular}

$\left(\mathrm{r}-\right.$ Werte; $\left.{ }^{\mathrm{a}} \mathrm{p}<0,05\right)$.

\section{Kardiopulmonale Funktionsparameter}

Die lungentransplantierten Patienten erreichten eine maximale Leistungsfähigkeit von lediglich 65 Watt bzw. $44 \%$ des altersund geschlechtsbezogenen Normwerts entsprechend einer $\mathrm{VO}_{2 \max }$ von $15,1 \mathrm{ml} \cdot \mathrm{min}^{-1} \cdot \mathrm{kg}^{-1}$. Frühere Untersuchungen im ersten Jahr nach Lungentransplantation beschrieben eine vergleichbar eingeschränkte körperliche Leistungsfähigkeit im ersten Jahr nach Lungentransplantation [13]. Auch wenn die körperliche Leistungsfähigkeit nach der Lungentransplantation im Vergleich zu Normalwerten absolut reduziert bleibt, so führt die Lungentransplantation doch zu einer 20 - 30-prozentigen Steigerung der körperlichen Leistungsfähigkeit [4].

Als Hauptursache der reduzierten körperlichen Leistungsfähigkeit Lungentransplantierter wird die Dysfunktion der Skelettmuskulatur beschrieben $[5,6,14]$. Untersuchungen bei Patienten mit schwerer chronisch obstruktiver Lungenerkrankung bzw. nach Lungentransplantation zeigten gleiche strukturelle Veränderungen ihrer Skelettmuskulatur [6,15-19]. In Muskelbiopsien wurde ein reduzierter Faseranteil der Typ-I-Muskulatur sowie eine Verminderung der oxidativen Kapazität der Mitochondrien nachgewiesen [6,15 - 19]. Weitere Studien mit Lungentransplantierten zeigten eine reduzierte Beinkraft und eine zu niedrigeren Belastungen hin reduzierte anaerobe Schwelle, während die ventilatorische Funktion kein leistungslimitierender Faktor mehr war $[5,14,20,21]$.
Die immunsuppressive Therapie führt zu einer zusätzlichen Behinderung der muskulären Rekonditionierung nach Transplantation. Aktuelle Untersuchungen über zelluläre Wirkmechanismen haben gezeigt, dass der Calcineurin-Inhibitor Cyclosporin A die Expression der langsamen schweren Myosinketten und den Muskelfasershift von schnellen, Typ-II- zu langsamen, Typ-I-Muskelfasern hemmt $[22,23]$. Somit wird vor allem die für die Alltagsbelastungen so wichtige aerobe Trainingsanpassung durch Cyclosporin A behindert. Kortikosteroide können eine muskuläre Atrophie und einen Kraftverlust aller Muskelfaseranteile verursachen. Bei Nierentransplantierten mit dauerhafter Cortisontherapie kam es zu einer Reduktion der Myofibrillen aller Muskelfasertypen [24].

Bei alltäglichen Belastungen werden die Auswirkungen der verminderten körperlichen Leistung unmittelbar deutlich: Die maximale Sauerstoffaufnahme der Lungentransplantierten betrug bei höchster Anstrengung durchschnittlich $1038 \mathrm{ml} \cdot \mathrm{min}^{-1}$ (Kontrollgruppe $2684 \mathrm{ml} \cdot \mathrm{min}^{-1}$ ). Für alltägliche Dauerbelastungen können ca. 50 - 75\% der maximalen Sauerstoffaufnahme eingesetzt werden, ohne frühzeitig zu ermüden, entsprechend bei den untersuchten lungentransplantierten Patienten ca. 500 bis $800 \mathrm{ml} \cdot \mathrm{min}^{-1}$ (Kontrollgruppe 1300 bis $1900 \mathrm{ml} \cdot \mathrm{min}^{-1}$ ).

Reinigungsarbeiten im Haushalt oder Tragen von Einkaufstaschen erfordern eine Sauerstoffaufnahme von etwa 800 bis $1400 \mathrm{ml} \cdot \mathrm{min}^{-1}$, Gartenarbeiten von 1100 bis $2750 \mathrm{ml} \cdot \mathrm{min}^{-1}$ [25]. Hieraus wird deutlich, dass übliche Freizeit- und Alltagsbelastungen für viele Patienten trotz eines gut funktionierenden Transplantats nur schwer möglich sind. Ein Ziel der Nachsorge sollte es daher sein, die körperliche Leistungsfähigkeit so zu steigern, dass Alltagsbelastungen ohne Überforderung und metabolische Ermüdung durchgeführt werden können. 


\section{Subjektive Einschätzung der Lebensqualität}

Die lungentransplantierten Patienten beschrieben eine annähernd gleich gute subjektive Lebensqualität wie die Kontrollgruppe bzw. das Kollektiv der PLC-Fragenbogen-Normierung. Obwohl die objektive körperliche Leistungsfähigkeit der Lungentransplantierten erheblich reduziert war (- 67\% im Vergleich zur Kontrollgruppe), bewerteten sie ihre Lebensqualität in der physischen Dimension nur tendenziell schlechter (-9\%) als die Kontrollgruppe.

Die Lungentransplantation führt zu einer dramatischen Verbesserung der von den Patienten empfundenen Lebensqualität. Vermeulen u. Mitarb. befragten in einer Längsschnittuntersuchung 28 Patienten vor und bis 55 Monate nach Lungentransplantation [26]. Die subjektive Lebensqualität sowie Toleranz von Alltagsbelastungen wurden 1 bis 3 Jahre nach Lungentransplantation als normal oder nur als gering reduziert bewertet. Im Langzeitverlauf verbesserten sich ebenfalls die psychiatrischen Indizes der Angst- und Depressionsskalen [26].

Der von uns in der Befragung der Lungentransplantierten angewandte, standardisierte Fragenbogen „Profil der Lebensqualität chronisch Kranker“ von Siegrist u. Mitarb. ergab vergleichbare Resultate in den drei Lebensqualitätsdimensionen wie andere, im englischen Sprachraum benutzte Fragebogen zur Erfassung der gesundheitsbezogenen Lebensqualität. Im Vergleich zur Kontrollgruppe Gesunder hatten Patienten nach Lungentransplantation z.T. leicht verminderte Scores in den physischen Skalen $[2,27,28]$. In den psychologischen wie in den sozialen Dimensionen wurden, wie auch in unserer Studie, keine oder nur geringfügige subjektive Einschränkungen beschrieben $[2,27,28]$.

\section{Korrelationen von subjektiver Lebensqualität mit objektiven Messgrößen}

Die vorliegende Untersuchung bestätigt, dass zwischen der Lebensqualität in der physischen Dimension und der gemessenen Leistungsfähigkeit im Stufentest eine signifikant positive Beziehung besteht. Die Einsekundenkapazität korreliert sowohl positiv mit der physischen Dimension der Lebensqualität und der körperlichen Leistungsfähigkeit. Dahingegen bestehen keine signifikanten Beziehungen zwischen psychischer bzw. sozialer Lebensqualität mit der Lungenfunktion bzw. der körperlichen Leistungsfähigkeit.

In einer früheren Studie mit Herztransplantierten konnte ebenfalls eine positiv signifikante Korrelation zwischen objektiver körperlicher Funktion und selbsteingeschätzter physischer Lebensqualität gezeigt werden [29]. Hier führte ein körperliches Trainingsprogramm zu einer Steigerung der körperlichen Leistungsfähigkeit und damit verbunden auch zu einer weiter verbesserten Selbsteinschätzung der Lebensqualität [30].

Basierend auf diesen Erkenntnissen bietet sich für die Transplantationsnachsorge neben einer indikationsbezogenen psychosozialen Betreuung auch ein systematisches körperliches Trainingsprogramm an. Ziele des Trainings sind eine Steigerung der peripher-muskulären Funktion, eine Vorbeugung gegen die durch Immunsuppressiva verursachte Myopathie [31], eine Erhöhung der körperlichen Belastbarkeit und damit eine Verbesserung der Lebensqualität und Alltagstoleranz.
Körperliche Trainingsprogramme sollten verstärkt auch im Stadium der chronischen Lungenerkrankung initiiert werden, um der peripher-muskulären Atrophie als Folge der Kortisontherapie und der körperlichen Schonung entgegen zu wirken.

\section{Schlussfolgerung}

Trotz guter Transplantatfunktion ist die körperliche Leistungsfähigkeit nach Lungentransplantation erheblich reduziert. Die Verminderung der Leistungsfähigkeit geht mit einer leicht eingeschränkten Lebensqualität in der physischen Dimension einher. Neben der rein medizinischen Langzeitnachsorge sollten daher körperliche Trainingsprogramme, ggf. in Kombination mit psychosozialer Betreuung angeboten werden, wenn die Verbesserung der Lebensqualität als ein Ziel der Lungentransplantation definiert wird.

\section{Literatur}

${ }^{1}$ Trulock EP, Edwards LB, Taylor DO et al. The registry of the international society for heart and lung transplantation: Twentieth offical adult lung and heart-lung transplant report - 2003. J Heart Lung Transplant 2003; 22: 625-635

${ }^{2}$ Cohen L, Littlefield C, Kelly P et al. Predictors of quality of life and adjustment after lung transplantation. Chest 1998; 113: 633-644

${ }^{3}$ TenVerget EM, Essink-Bot ML, Geertsma A et al. The effect of lung transplantation on health-related quality of life. Chest 1998; 113: $358-364$

${ }^{4}$ Schwaiblmair M, Reichenspurner $\mathrm{H}$, Müller $\mathrm{C}$ et al. Cardiopulmonary exercise testing before and after lung and heart-lung transplantation. Am J Respir Crit Care Med 1999; 159: 1277-1283

${ }^{5}$ Lands LC, Smountas AA, Mesiano G et al. Maximal exercise capacity and peripheral skeletal muscle function following lung transplantation. J Heart Lung Transplant 1999; 18: $113-120$

${ }^{6}$ Wang XN, Williams TJ, McKenna MJ et al. Skeletal muscle oxidative capacity, fiber type, and metabolits after lung transplantation. Am J Respir Crit Care Med 1999; 160: 57-63

${ }^{7}$ Lanuza ML, Lefaiver CA, Farcas GA. Research on the quality of life of lung transplant candidates and recipients: an integrated review. Heart Lung 2000; 29: 180-195

8 Parízková J, Goldstein H. A comparison of skinfold measurements using Best and Harpenden calipers. Hum Biol 1970; 42: 436- 441

${ }^{9}$ Siegrist J, Broer M, Junge A. Profil der Lebensqualität chronisch Kranker. Göttingen: Beltz Test, 1996: 23 - 46

${ }^{10}$ Künsebeck HW, Körber J, Freyberger H. Quality of life in patients with inflammatory bowel disease. Psychotherapy and psychosomatics 1990; 54: $110-116$

${ }^{11}$ Borg GA. Psychophysical bases of perceived exertion. Med Sci Sports Exerc 1982; 14: $377-381$

${ }^{12}$ Laubach W, Schröder C, Siegrist J et al. Normierung der Skalen „Profil der Lebensqualität Chronisch Kranker“ an einer repräsentativen deutschen Stichprobe. ZDDP 2001; 22: 100-110

13 Oelberg DA, Systrom DM, Markowitz DH et al. Exercise performance in cystic fibrosis before and after bilateral lung transplantation. J Heart Lung Transplant 1998; 17: 1104-1112

14 Tridel GB, Girgis R, Fishman RS et al. Metabolics myopathy as a cause of the exercise limitation in lung transplant recipients. J Heart Lung Transplant 1998; 17: 1231-1237

15 Jakobsson P, Jorfeldt L, Brundin A. Skeletal muscle metabolites and fibre types in patients with advanced chronic obstructive pulmonary disease (COPD) with and without chronic respiratory failure. Eur Respir J 1990; 3: 192 - 196

${ }^{16}$ Whittom F, Jobin J, Simard PM et al. Histochemical and morphological characteristics of the vastus lateralis in COPD patients. Med Sci Sports Exerc 1998; 30: 1467-1474

${ }_{17}$ Meer K De, Jeneson JAL, Gulmans AM et al. Efficiency of oxidative work performance of skeletal muscle in patients with cystic fibrosis. Thorax 1995; 50: $980-983$ 
${ }^{18}$ Evans AB, Al-Himyary AJ, Hrovat MI et al. Abnormal skeletal muscle oxidative capacity after lung transplantation by 31P-MRS. Am J Respir Crit Care Med 1997; 155: 615-621

${ }^{19}$ Jakobsson P, Jorfeldt L, Henriksson J. Metabolic enzyme activity in the quadrizeps femoris muscle in patients with severe chronic obstructive pulmonary disease. Am J Respir Crit Care Med 1995; 151: 374 - 377

${ }^{20}$ Levy RD, Ernst P, Levine SM et al. Exercise performance after lung transplantation. J Heart Lung Transplant 1993; 12: 27 - 33

${ }^{21}$ Systrom DM, Pappagianopoulos P, Fishman RS et al. Determinants of abnormal maximum oxygen uptake after lung transplantation for chronic obstructive pulmonary disease. J Heart Lung Transplant 1998; 17: $1220-1230$

22 Meißner JD, Gros G, Scheibe RJ et al. Calcineurin regulates slow myosin, but not fast myosin or metabolic enzymes, during fast-to-slow transformation in rabbit skeletal muscle cell culture. J Physiol 2001; 533: $215-226$

${ }^{23}$ Kubis HP, Scheibe RJ, Meissner JD et al. Fast-to-slow transformation and nuclear import/export kinetics of the transcription factor NFATc1 during electrostimulation of rabbit muscle cells in culture. J Physiol 2002; 541: 835-847

${ }^{24}$ Horber FF, Hoppeler H, Herren D et al. Altered skeletal muscle ultrastructure in renal transplant patients on prednisone. Kidney Int 1986; 30: $411-416$

${ }^{25}$ McArdle WD, Katch FI, Katch VI (Hrsg). Exercise Physiology: energy, nutrition, and human performance. 4th ed. Baltimore: Williams and Wilkins, 1996: 769-781

${ }^{26}$ Vermeulen KM, Ouwens JP, Bij W van der et al. Long-term quality of life in patients surviving at least 55 months after lung transplantation. Gen Hosp Psychiatry 2003; 25: 95-102

${ }^{27}$ Stavem K, Bjortuft O, Lund MB et al. Health-related quality of life in lung transplanat candidates and recipients. Respiration 2000; 67: $159-165$

${ }^{28}$ Gross CR, Savik K, Bolman RM et al. Long-term health status and quality of life outcomes of lung transplanat recipients. Chest 1995; 108: $1587-1593$

${ }^{29}$ Tegtbur U, Pethig K, Jung K et al. Lebensqualität im Langzeitverlauf nach Herztransplantation. Z Kardiol 2003; 92: 660-667

${ }^{30}$ Tegtbur U, Pethig K, Machold H et al. Exercise Training and Quality of Life in Long-term Treatment after Heart Transplantation. Circulation 2001; 104 (Suppl. 2): 646

${ }^{31}$ Horber FF, Scheidegger JR, Grunig BE et al. Evidence that prednisoneinduced myopathy is reversed by physical training. J Clin Endocrinol Metab 1985; 61: 83-88
Erratum zur Tagung der Sektion Pathophysiologie und Aerosolmedizin in der DGP, Berlin, 21.-22. November 2003. Pneumologie 2003; 57: $763-770$

Bei der Publikation der Abstracts zur Herbsttagung der Sektion wurde der Beitrag von Frau Dr. Hilgendorff versehentlich nicht gedruckt.

Stellenwert der multiplen Okklusionstechnik zur Bestimmung der Lungendehnbarkeit im Rahmen der Lungenfunktionsdiagnostik bei Säuglingen mit bronchopulmonaler Dysplasie.

Anne Hilgendorff, G. Hüls, L. Gortner, H. Lindemann

Zentrum für Kinderheilkunde und Jugendmedizin, Universitätsklinik Gießen, Deutschland

Die bronchopulmonale Dysplasie (BPD) bleibt eine wichtige Komplikation im Langzeitverlauf als Folge eines Atemnotsyndroms bei Frühgeborenen. Neben definierten klinischen und radiologischen Kriterien, die zur Diagnosestellung herangezogen werden, fehlen jedoch hinreichend aussagekräftige Variablen zur Differenzierung der pulmonalen Veränderung. Zu ihrer Etablierung wurden frühgeborene Säuglinge (FG) mit der Diagnose einer BPD, definiert als Sauerstoffbedarf am 28. Lebenstag, prospektiv einer Lungenfunktionsdiagnostik zugeführt. Zum errechneten Geburtstermin konnten 8 FG mit der Diagnose einer BPD im Master Screen Baby Body (Fa. Jaeger, Hoechberg, Deutschland) bodyplethysmografisch untersucht werden. Es wurden Atemwegswiderstand und funktionelle Residualkapazität $\left(\mathrm{FRC}_{\text {box. }}\right.$ ) ermittelt. Zur näheren Charakterisierung der pulmonalen Schädigung erfolgte zusätzlich die Messung der Lungencompliance bezogen auf die Atemruhelage $\left(\mathrm{C}_{\mathrm{L}} / \mathrm{FRC}_{\mathrm{box}}\right)$ mit Hilfe der multiplen Okklusionstechnik (MOT). Die Messungen erforderten eine milde Sedierung der Patienten. Trotz eines immer unauffälligen Auskultationsbefundes zeigte sich bei vier FG ein mittelgradig bis deutlich erhöhter exspiratorischer Atemwegswiderstand. Eine Überblähung fand sich bei drei Patienten. Bei vier FG ließ sich eine deutliche Erniedrigung des $\mathrm{FRC}_{\mathrm{box}}$ nachweisen in Zusammenhang mit fehlenden bis nur milden Veränderungen im Sinne einer obstruktiven Ventilationsstörung. Sowohl die $\mathrm{C}_{\mathrm{L}} /$ $\mathrm{FRC}_{\mathrm{box}}$ als auch das Vorliegen obstruktiver Ventilationsstörungen oder Bestehen eines Volumenmangels zeigte eine tendentielle Abhängigkeit vom Gestationsalter. Fazit: Zur Einschätzung des Anteils obstruktiver und restriktiver Ventilationsstörungen am klinischen Bild der BPD erscheint die differenzierte Lungenfunktionsdiagnostik, für die die MOT eine wichtige Ergänzung und Erweiterung darstellt, gut geeignet. 